\title{
TIME BETWEEN DIAGNOSIS AND BEGIN OF THE TREATMENT IN A PUBLIC BRAZILIAN HOSPITAL IN SÃO PAULO
}

\author{
André Mattar¹, Jorge Y. Shida¹, Roberto Hegg¹, Felipe A. Cavagna¹, Ana Luiza A. Faria1, Luiz H. Gebrim¹ \\ 'Women's Reference Center, Hospital Pérola Byington - São Paulo (SP), Brazil.
}

Objectives: Breast cancer affects about 59,000 Brazilian women annually according to data from INCA 2018 (National Health Institute) and almost 55\% of patients are diagnosed in advanced stages. In most Brazilian câncer centers the treatment is made only with the definitive diagnosis (biopsy). It can lead to a delay of 5 to 8 months and may even take longer. On November 22, 2012, a federal law established in 60 days the maximum period between the diagnosis and the first treatment of any malignant disease. Pérola Byington Hospital is one of the most important cancer centers specialized Breast Cancer treatment in Brazil. In 2005 a model of visits based on quick diagnosis and treatment was implemented named CARE (Centro de Alta Resolutividade - High Resolutivity Center). This model is based in "One Stop Clinic" approach contemplates the integration between the professionals and the physical space in the same physical area. In this model a trained physician can have the results in the same day of mammography and ultrasonography. Biopsy is performed in the same day and after 2 or 3 weeks our pathologists trained in mammary pathology can give us the results with the immunohistochemistry analyses. With this approach, we have been able to optimize resources and gain the agility to diagnose breast cancer suspects, whether palpable or not. Many breast cancer cases require primary (neoadjuvant) treatments since many diagnoses were already made in late phases. In 2016, there was a need for integration with the clinical oncology service, allowing a clinical visit by the oncologist in the same day and the possibility to initiate the treatment between 7 to 10 days. This model based on a single visit requires an integrated and trained multidisciplinary team, not only in the treatment of the disease but also training in this model of approach. The idea of this model was born due to the observation that patients in Brazil usually spent a long time in lines to the first visit and had to wait long time to perform the specific exams (mammography/ultrasound) and then a long time to have an evaluation with the specialist and more time spend to undergo invasive diagnostic procedures. Usually patients had to return 3 or 4 times to have their diagnosis made and this process could take 2 or 3 moths to start the treatment. With our approach we have been able to reduce the waiting time substantially, since all the tests are made on the same day (including biopsy), anticipating the diagnosis and only for this reason reducing the number of advanced cases. Since the prognosis is closely linked to the stage, this approach can substantially increase disease cure rates. Our Center annually treats about a thousand new cases of breast cancer and about 1400 surgical procedures are required. We have been spreading this idea promoting workshops, continued medical education; training courses in mammography and ultrasound diagnosis and medical training in percutaneous biopsies, both guided by ultrasonography and stereotactic. Our mission is to promote humanized treatment and achieve early diagnosis and timely treatment to achieve a better prognosis. Methodology: This study was performed at the Pérola Byington Hospital evaluating patients treated at our hospital from 2011 to 2017. We have reviewed the cases diagnosed with breast cancer at the hospital and the date of diagnosis and the beginning of treatment were evaluated, being it surgical or performed chemotherapy. Results: In this period 6,488 cases of breast cancer were included in our evaluation. The mean between the initial histological or cytologic diagnosis and the beginning of the treatment was calculated in each year: In $201163.82 \%$ of our cases had their first treatment beyond 60 days. In 2017 most of our patients were treated in at least 60 days $(67.23 \%)$. We know that the mean can be very variable if one of the numbers is too large or too small and because of that we have been able to median between the diagnosis and the first treatment. In 2011 the median was 76, in 2012 was 83 and this number was going down year by year and in 2017 the median is 53 days. Conclusions: In our Hospital the "One Stop Clinic" approach have changed the way of treating the patients allowing more agility in the treatment of breast cancer including quick diagnosis and initiation of treatment as soon as possible. 